\title{
Analysis of Human Electrocardiogram for Biometric Recognition Using Analytic and AR Modeling Extracted Parameters
}

\author{
Branislav Vuksanovic \\ Faculty of technology/School of engineering \\ University of Portsmouth \\ Portsmouth, PO1 2UP, United Kingdom \\ Mustafa Alhamdi \\ Faculty of technology/School of engineering \\ University of Portsmouth \\ Portsmouth, PO1 2UP, United Kingdom
}

branisalv.vuksanovic@port.ac.uk

mustafa.alhamdi@port.ac.uk

\begin{abstract}
The electrocardiograph (ECG) contains cardiac features unique to each individual. By analyzing ECG, it should therefore be possible not only to detect the rate and consistency of heartbeats but to also extract other signal features in order to identify ECG records belonging to individual subjects. In this paper, a new approach for automatic analysis of single lead ECG for human recognition is proposed and evaluated. Eighteen temporal, amplitude, width and autoregressive (AR) model parameters are extracted from each ECG beat and classified in order to identify each individual. Proposed system uses pre-processing stage to decrease the effects of noise and other unwanted artifacts usually present in raw ECG data. Following pre-processing steps, ECG stream is partitioned into separate windows where each window includes single beat of ECG signal. Window estimation is based on the localization of the R peaks in the ECG stream that detected by Filter bank method for QRS complex detection. ECG features - temporal, amplitude and AR coefficients are then extracted and used as an input to K-nn and SVM classification algorithms in order to identify the individual subjects and beats. Signal pre-processing techniques, applied feature extraction methods and some intermediate and final classification results are presented in this paper.
\end{abstract}

Keywords: ECG Biometric, Filtering, QRS Detection, AR Model, Extraction and Classification.

\section{INTRODUCTION}

Biometric recognition provides authentication by identifying each individual based on the biological and physiological signal characteristics. A number of identification methods have been investigated in the last decades [1], using physical features such as finger prints, face images [2] and biological signal behavior such as electrocardiograph (ECG) [3]. Analysis of ECG signals as a biological tool for individual recognition has become an active research field in the past two decades [4][5]. Validity of using ECG as a biometric tool is supported by the fact that each ECG signal has certain unique features [6] which can be used to distinguish it from other ECG signals. Differences between ECG signals are usually caused by the variability of heart position and orientation relative to the ribs (the ribs being the reference clinically used to place the precordial electrodes on body), which are highly variable among different persons. Other differences can be related to body habitus [7], sex, age, length, and weight of the subjects.

The ECG signal classification is usually considered in the light of selection, extraction and classification of extracted features. High recognition rate has been achieved with the approach based on the autocorrelation (AC) in conjunction with discrete cosine transform (DCT) [3]. Proposed method does not require any waveform or fiducial point detections but AC and DCT can be computationally demanding and require long ECG records for each subject or individual to 
identify them successfully. A method known as Pulse Active Width (PAW) is implemented on ECG for biometric authentication [8]. The results of this approach have indicated that PAW yields equivalent performance in terms of accuracy compared to conventional temporal and amplitude feature extraction methods. However, PAW is a complex signal processing technique which requires powerful digital signal processors to overcome the time delay.

The electrocardiogram is an emerging biometric modality that has seen about 13 years of development reported in peer-reviewed literature [9], and as such deserves a systematic review and discussion of the associated methods and findings. In particular, the categorization of methodologies in ECG based biometry relies on the feature extraction and classification schemes. Finally, comparative analysis is presented to estimate performance of this approach of ECG biometric system. The comparative study includes the cases where training and testing data sets come from the same and different partition of ECG signal measured on different days. Recently, cardiovascular signals have been studied for use in identity recognition problems using electrocardiography [10], [11] and [12].

A number of different techniques have been implemented in the past years to design ECG classification system. Mahmoodabadi [13] described an approach for ECG feature extraction which utilizes multi-resolution wavelet transform for ECG feature extraction. In this desired study, the ECG signals from modified lead II were chosen for processing. Experimental results showed that proposed approach for ECG feature extraction achieved accuracy of up to $98 \%$ to classify ECG signals. A mathematical morphology based on Kohonen self-organizing maps (SOM) and Learning Vector Quantization (LVQ) to design ECG feature extraction was proposed by Tadejko and Rakowski [14]. The main focus of this work is to evaluate the classification performance of an automatic classifier of the ECG designed to detect abnormal beats using new concept of feature extraction stage. The accuracy or correct classification rate of this method is $94 \%$. An automatic extraction of both time interval and morphological features from ECG to classify ECGs into normal and arrhythmic was described by Alexak [15]. The method utilized the combination of artificial neural networks (ANN) and Linear Discriminant Analysis (LDA) techniques for feature extraction. The average accuracy of this method is about $85 \%$. A new ECG pattern classification model based on a generic feature extraction method is presented in [16]. Correct classification rate is $88 \%$ for this method. Work described in [17] presents an approach to ECG signal filtering and classification system using HMM. Achieved accuracy of this method is up to $95 \%$.

In this paper, a new approach is developed and evaluated for automatic analysis of single lead electrocardiogram (ECG) for human recognition and individual identification. This approach is depended on analytic (Amplitude, Time and Width) and modeling (AR) features extracted from the ECG beat. Obtained results indicate high level of accuracy and shorter processing needed to identify the individuals. Eighteen analytic and modeling features are extracted to identify individuals. $\mathrm{K}$ nearest neighbor (k-nn) classification and Support Vector Machine (SVM) algorithms are applied in order to classify those features and evaluate the proposed approach. ECG feature selection and extraction using AR modeling has recently been used [18] resulting in accurate classification of various arrhythmia and ventricular arrhythmia conditions.

The remainder of this paper is organized as follows. Section 2 gives a brief description of the techniques used in the pre-processing phase to clean ECG signals of noise and other artefacts. Section 3 provides a review of QRS detection method used in this work. Feature selection and extraction methods are discussed in Section 4 whilst Section 5 contains experimental results and discussion of those results. Conclusions are presented in Section 6.

\section{PRE-PRROCESSING}

ECG signal is recorded by attaching electrodes to different places on the skin, such as chest, legs, arms and neck [19]. Collected ECG data usually contain noise components of low-frequency caused by driftline wonder and high frequency components caused by power line interferences [20]. The presence of noise will corrupt the signal and make the feature extraction and 
classification process more difficult and less accurate. Many research papers have discussed the removal of noise and power line interference from the ECG signals. In [21] a non linear adaptive method to eliminate power line interference from the ECG signals is presented. The wavelet coefficient threshold based hyper shrinkage function was used in [22] to detrend the raw ECG signals. In [23] a simplified lattice based adaptive IIR notch filter has been suggested to remove power line interference. Digital FIR filters has been used for the power line noise reduction [24].

In this work, method proposed in [25] has been applied in order to decrease the effect of driftline wonder and preserve the information about the physiology of an individual's ECG. The raw ECG signals are first downsampled to the lowest sampling frequency of the ECG signals from the set $\left(1000 \mathrm{~Hz}\right.$ in this case) and then filtered through the $6^{\text {th }}$ order high pass Butterworth filter. Figures $1 \mathrm{a}$ and $1 \mathrm{~b}$ illustrate the performance of the applied Butterworth filter in the pre-processing stage of the proposed system. The output of the Butterworth filter is then passed through power line interferences filer. Here, another Butterworth filter $-10^{\text {th }}$ order low pass type is used to remove high frequency components and noise. Figures $1 \mathrm{c}$ and $1 \mathrm{~d}$ show the signals after the power line interference filtering stage.

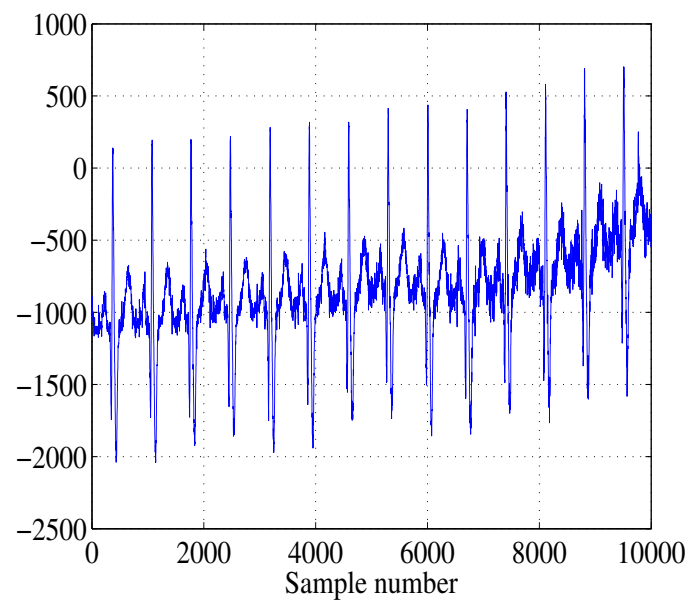

a. Original ECG signal

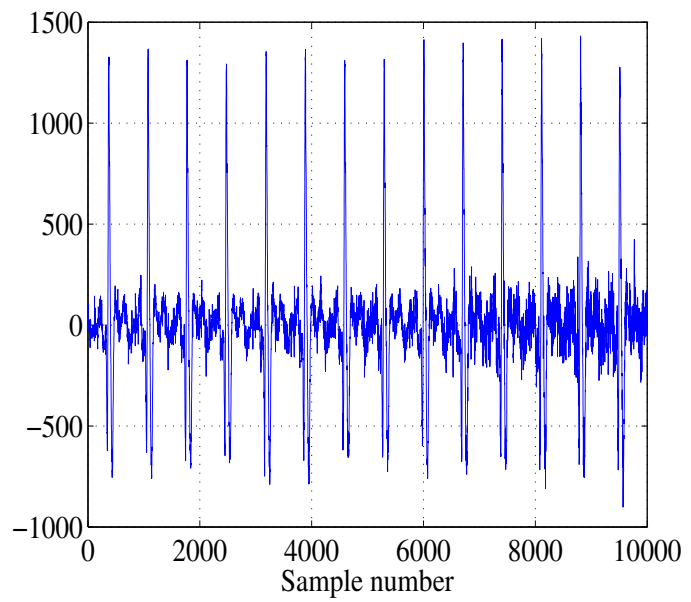

c. Original ECG signal

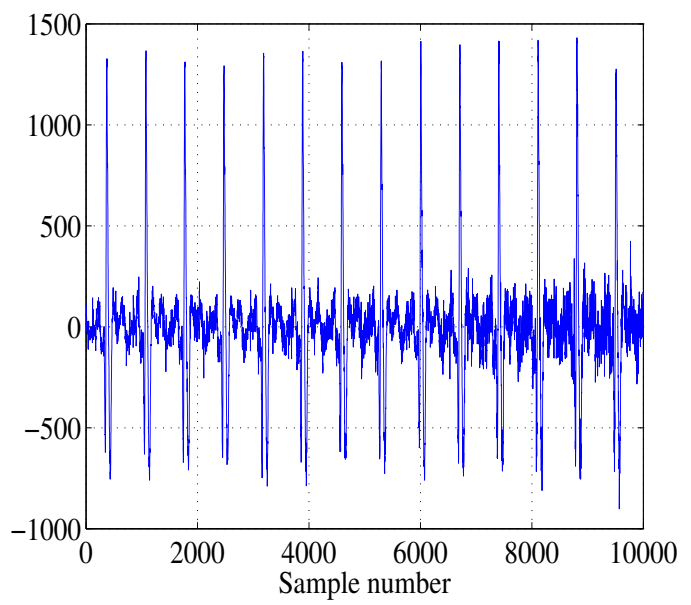

b. ECG signal after passing through the driftline wonder filtering stage

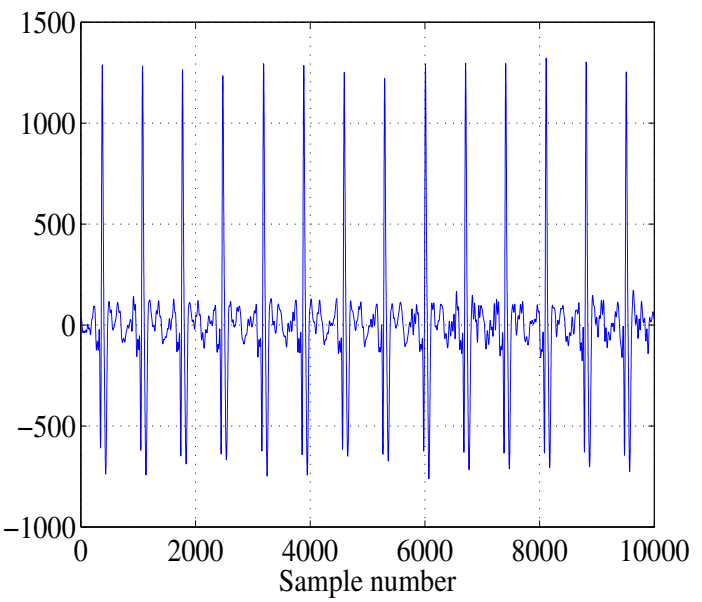

d. ECG signal after passing through the power line interference removal filtering stage

FIGURE 1: Driftline wonder and power line interference filtering stages. 


\section{QRS DETECTION PHASE}

The QRS complex is the most important feature of the ECG signal. Without the accurate knowledge of the QRS complex location, $\mathrm{P}$ and $\mathrm{T}$ waves are hard to detect and distinguish from each other. Most of the QRS detection methods rely on filtering stage followed by averaging and thresholding [26] in order to distinguish between noise signal and the QRS complex. Threshold value is usually chosen according to the peak height or peak location of ECG signal [27]. A research paper has investigated the problem of accurate QRS complex detection depending on the applications of wavelet filter banks [28]. The short-time Fourier transform (STFT) was also employed after ECG filtering stage in order to detect QRS complex [29].

In this work, Filter bank method employs a bank of linear phase filters to decompose the ECG signal into subbands with uniform frequency bandwidths in order to account for the ECG signal energy distribution in the frequency domain [28]. Processing can be performed on each subband independently. The filterbank used to analyse ECG signals contains $M$ analysis and $M$ synthesis filters, each of length $L$ and is used to produce the subband signals $U_{1}(z)$ by filtering the input signal $\mathrm{X}(z)$ :

$$
\mathrm{U}_{\mathrm{l}}(z)=\mathrm{H}_{\mathrm{l}}(z) \mathrm{X}(z) \quad \mathrm{l}=0,1, \ldots, M-1
$$

where $\mathrm{H}_{1}(z)$ represents the transfer function of each bandpass filter. The set of synthesis filters can then combine the processed subbands to result in a processed version of the input signal. Thus, a filter bank-based algorithm involves decomposing a signal into frequency subbands, processing these subbands according to the application at hand, and then sometimes reconstructing the processed subbands. Many scenarios deal with signals which contain specific energy distributions in the frequency domain. For example, with regard to the ECG, a significant proportion of the energy from the QRS complex extends to a frequency of $40 \mathrm{~Hz}$, and even more if the $\mathrm{Q}, \mathrm{R}$, and $\mathrm{S}$ waves have very sharp morphologies. The $\mathrm{P}$ and $\mathrm{T}$ waves, in general, have a significant proportion of their energy only up to $10 \mathrm{~Hz}$.

The effective bandwidth of $\mathrm{U}_{1}(z)$ is $\frac{\pi}{M}$ and, thus, it can be downsampled to reduce the total rate. The downsampling process keeps one sample out of $M$ samples. The downsampled signal $\mathrm{W}_{\mathrm{l}}(z)$ is:

$$
\mathrm{W}_{\mathrm{l}}(z)=\frac{1}{M} \sum_{\mathrm{k}=0}^{M-1} \mathrm{U}_{\mathrm{l}}\left(Z^{\frac{1}{M}} W^{k}\right)
$$

where $\mathrm{W}=\mathrm{e}^{-\mathrm{j}\left(\frac{2 \pi}{M}\right)}$. The subbands $\mathrm{U}_{\mathrm{l}}(z)$ and $\mathrm{W}_{\mathrm{l}}(z)$ are bandpassed versions of the input $\mathrm{X}(z)$ and also $\mathrm{W}_{1}(z)$ has a lower rate than $\mathrm{U}_{1}(z)$. The filtering process can be efficiently done at $\frac{1}{M}$, the input rate by taking advantage of the downsampling. This process is referred to as the polyphase implementation and contributes to the computational efficiency of the filter bank-based algorithm. A variety of features, indicative of the QRS complex have been extracted from the signal. Figure 2 shows QRS detection of this method by combining the subbands of interest. For example, feature $P_{1}$ corresponding to the energy in those subbands 1,2 and 3, can be computed as:

$$
P_{1}=\sum_{l=1}^{3}\left|W_{1}(z)\right|
$$




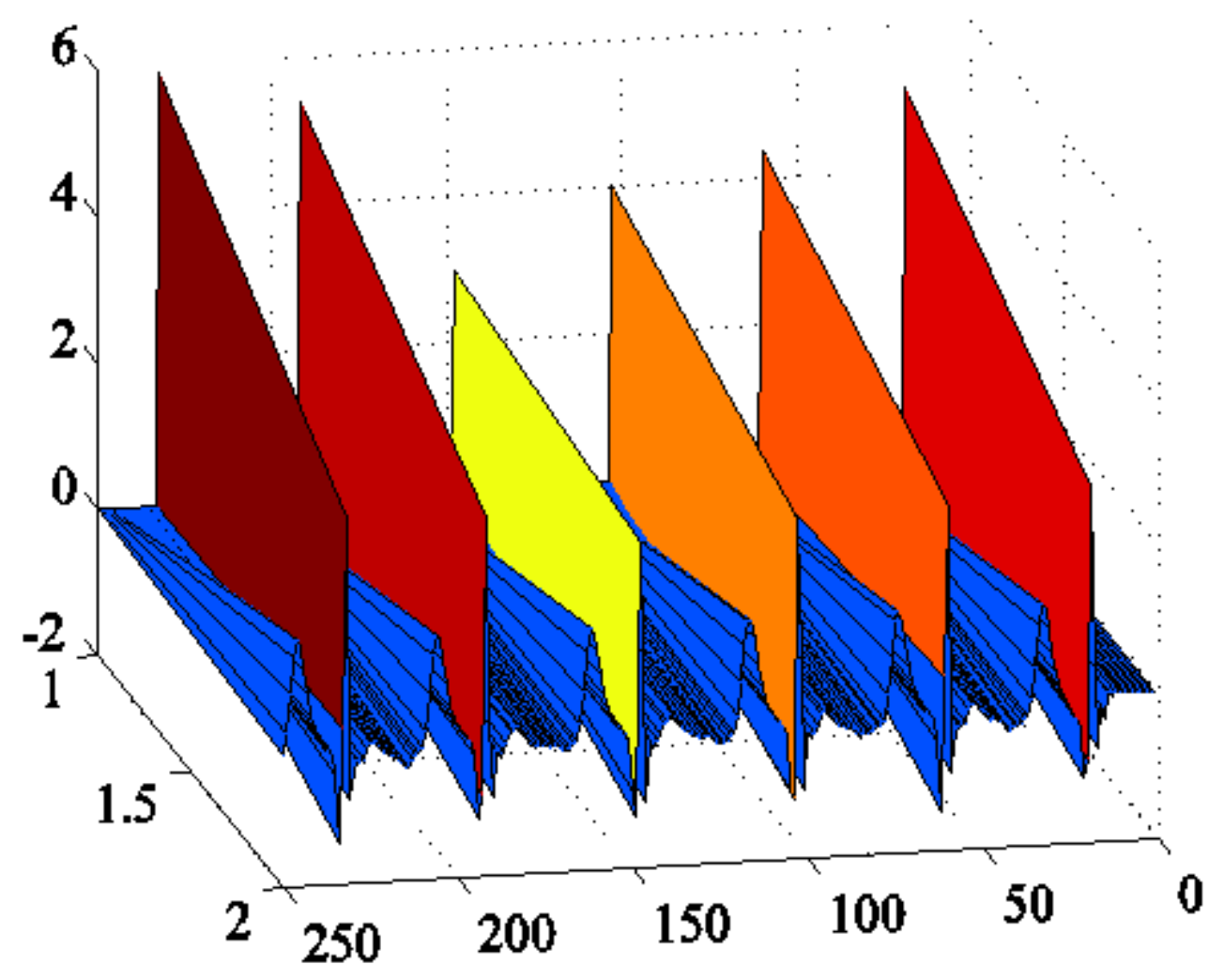

FIGURE 2: Filtered ECG signal with detected QRS complexes $\left(^{*}\right)$ using filter bank method.

In the ECG signals, $P$ and T waves occur before and after the QRS complex respectively. The frequency components of QRS complex range between $10 \mathrm{~Hz}$ and $25 \mathrm{~Hz}$. In this work, QRS detection plays an important stage as it is used to calculate RR interval of ECG stream, the main indicator for the window estimation process. Window estimation is applied in order to extract windows such that each window contains single beat of ECG windows. If $X(t)$ is an ECG stream of beats, window of single ECG beat is estimated based on the RR interval between any two consecutive R peaks "*” detected from the ECG stream and shafted by half of the RR interval.

$$
\mathrm{W}_{B}(t)=\sum_{\mathrm{i}=1}^{B} \mathrm{X}\left(t+0.5 R R_{i}\right)+\mathrm{X}\left(t-0.5 R R_{i}\right)
$$

where $R R$ is the interval between two consecutive R beats, $\mathrm{W}_{B}(t)$ is a window that contain single beat and $B$ is the number of beats in the ECG stream. Figure 3 shows four different samples of window estimation process, each window is plotted in $3 \mathrm{D}$ view and contains only one beat. 

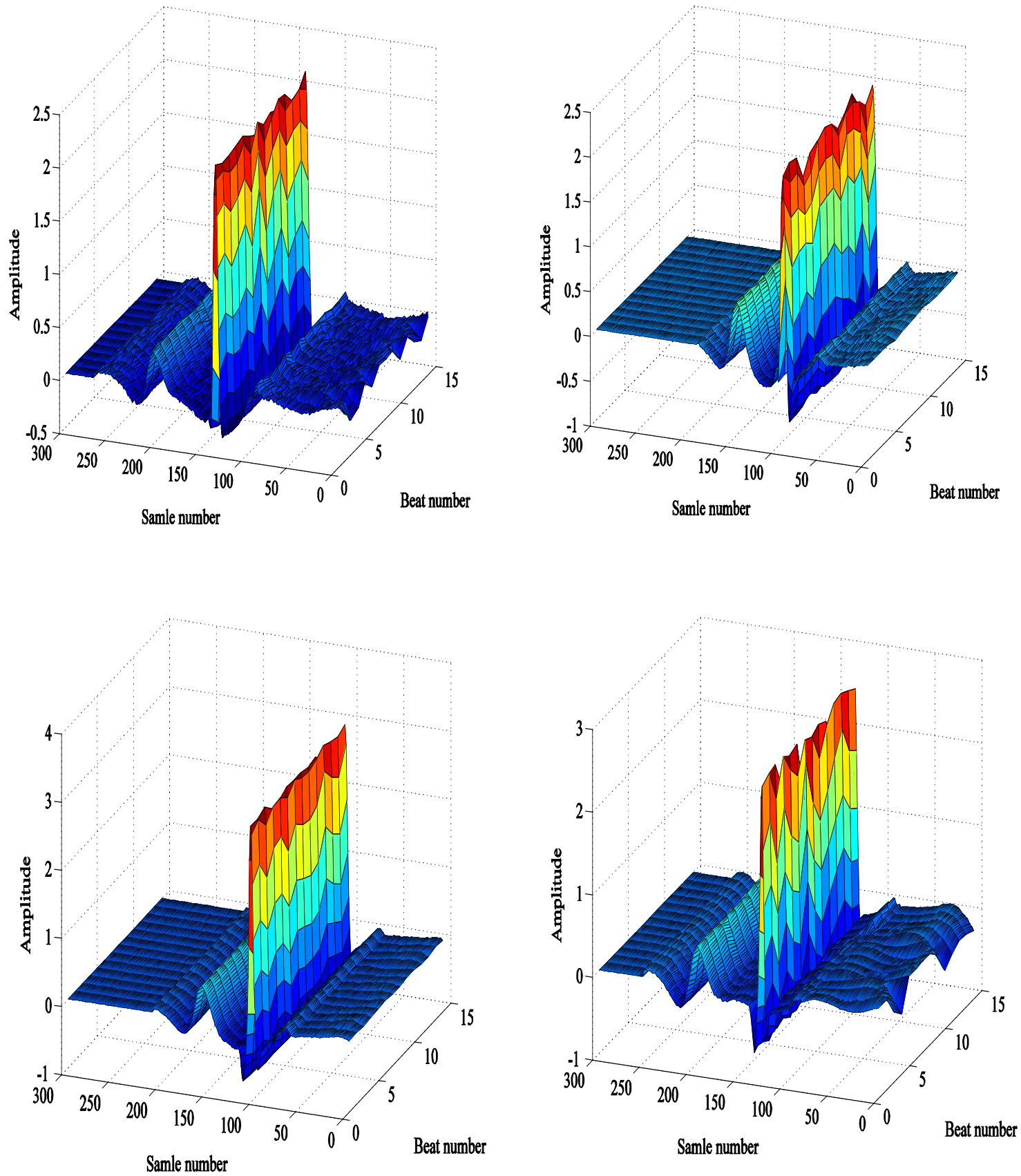

FIGURE 3: Window estimation for four subjects from MIT/BIH database.

\section{METHODOLOGY}

Biometrics-based human identification is essentially a subject and beat recognition problem which involves pre-processing, feature extraction and classification stages. ECG feature extraction plays a significant role in diagnosing most of the cardiac diseases. Feature extraction scheme proposed in this work uses amplitudes, time intervals, width and AR parameters of the ECG signal for subsequent analysis and identification. For the purpose of comparative study, the system proposed in this work follows the procedure of extracting eighteen features from each ECG beat listed in Table 1. 


\begin{tabular}{|c|c|c|c|c|c|}
\hline \multirow{9}{*}{ Analytic } & Temporal & $P Q$ & $\begin{array}{c}\text { Time interval between } P \text { and } \\
Q \text { waves. }\end{array}$ & PS & $\begin{array}{l}\text { Time interval between } \\
\mathrm{P} \text { and } \mathrm{S} \text { waves. }\end{array}$ \\
\hline & & PR & $\begin{array}{c}\text { Time interval between } P \text { and } \\
R \text { waves. }\end{array}$ & TS & $\begin{array}{l}\text { Time interval between } \mathrm{T} \\
\text { and } \mathrm{S} \text { waves. }\end{array}$ \\
\hline & & $\mathrm{TQ}$ & $\begin{array}{c}\text { Time interval between } T \text { and } \\
Q \text { waves. }\end{array}$ & TR & $\begin{array}{c}\text { Time interval between } \mathrm{T} \\
\text { and } \mathrm{R} \text { waves. }\end{array}$ \\
\hline & & PT & $\begin{array}{c}\text { Time interval between } \mathrm{P} \text { and } \\
\mathrm{T} \text { waves. }\end{array}$ & & \\
\hline & Amplitude & $P$ & Amplitude height of $P$ wave. & $\mathrm{T}$ & $\begin{array}{c}\text { Amplitude height of } \mathrm{T} \\
\text { wave. }\end{array}$ \\
\hline & & $S$ & Amplitude height of S wave. & $\mathrm{R}$ & $\begin{array}{c}\text { Amplitude height of } \mathrm{R} \\
\text { wave. }\end{array}$ \\
\hline & & Q & Amplitude height of $Q$ wave. & & \\
\hline & Width & QRS & Width in time of QRS. & $\mathrm{T}$ & Width in time of $\mathrm{T}$ wave. \\
\hline & & $\mathrm{P}$ & Width in time of $\mathrm{P}$ wave. & & \\
\hline \multirow[t]{2}{*}{ Modeling } & $\begin{array}{l}\text { AR } \\
\text { coefficients }\end{array}$ & $a_{1}$ & First coefficient of AR model. & $a_{3}$ & $\begin{array}{c}\text { Third coefficient of AR } \\
\text { model. }\end{array}$ \\
\hline & & $a_{2}$ & $\begin{array}{l}\text { Second coefficient of AR } \\
\text { model. }\end{array}$ & & \\
\hline
\end{tabular}

TABLE 1: List of extracted features.

\subsection{Analytic Features}

Analytic features are usually being extracted by analysing and localizing certain fiducial points from the ECG signals as shown in Figure 4. In order to extract analytic features of ECG window or single beat, procedure similar to [30] is followed. As the R peak is already detected using QRS detection, $\mathrm{Q}, \mathrm{S}, \mathrm{P}$ and $\mathrm{T}$ amplitudes are localized by finding local positive and negative points separately around $R$ positions of each window. As example, the maximum positive to the left of ECG beat in Figure 4 is the $P$ wave amplitude, and the maximum negative to the left is $Q$ wave amplitude. Same procedure is followed to find $T$ and $S$ amplitudes on the right side. Previously proposed methods [10], [11] and [12] for ECG-based identity recognition use temporal attributes as well as amplitude distances between detected fiducial points. In this work temporal, amplitude and width distances between indicated points have been fitted into k-nn and SVM classification algorithms to find subject and beat classification rate. Temporal and width extracted parameters are calculated using fiducial points in Figure 4, e.g. the time interval between $\mathrm{P}_{\text {start }}$ and $\mathrm{P}_{\text {end }}$ is the $P$ wave width. Similar method is used to estimate QRS complex width, the interval between $Q_{\text {start }}$ and $S_{\text {end }}$ and so on to estimate all the analytic features in Table 1. 


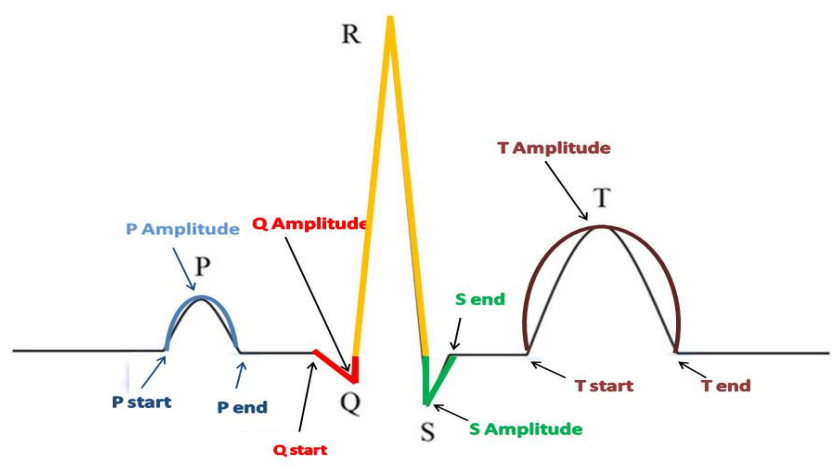

FIGURE 4: Fiducial points of ECG signal.

\subsection{Modeling Features}

In addition to analytic ECG features described in the previous section, proposed system uses modeling features obtained through the modeling of two or more successive ECG beats. To achieve this, a discrete form of an autoregressive (AR) signal model of order $p, \operatorname{AR}(p)$, is applied [31] to model the measured ECG signal. The estimated coefficients are then added to a feature set used in the final, classification stage of the proposed system.

Using the proposed AR model, a sampled signal sequence $y(n)$ can be represented by the relationship:

$$
\mathrm{y}(n)=a_{1} \mathrm{y}(n-1)+a_{2} \mathrm{y}(n-2)+\cdots+a_{p} \mathrm{y}(n-p)+\varepsilon(n)
$$

where $a_{k}(k=1,2, \ldots, p)$ are the model coefficients used in the classification process, the $\varepsilon(n)$ is a white noise series and $\sigma^{2}$ is the innovation process with zero mean and variance. Accurate modeling of two successive beats from ECG signal can be achieved using a $3^{\text {rd }}$ order AR model as described in [18]. Individual section of a pre-processed ECG signal in red and the corresponding $\mathrm{AR}(3)$ model in blue are shown in Figure 5. High accuracy of obtained AR models can be observed from this plot. $a_{1}, a_{2}$ and $a_{3}$ AR model coefficients are extracted from each beat of ECG stream and fitted into k-nn and SVM with analytic features in the classification process.

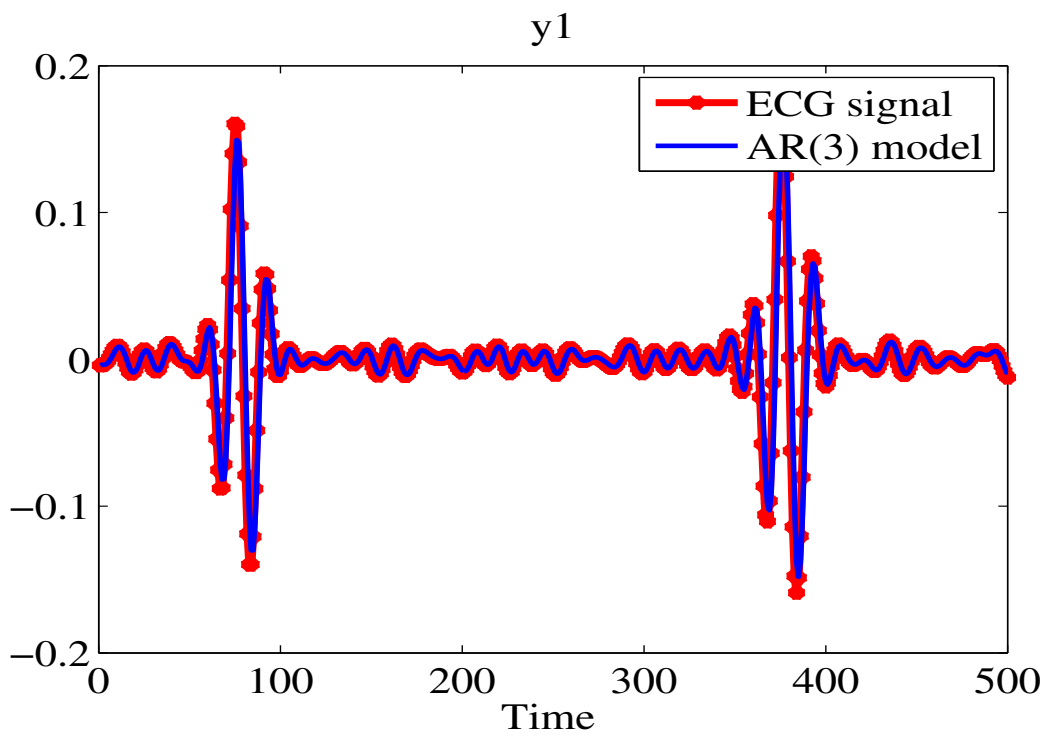

FIGURE 5: AR model and original ECG signal. 


\section{EXPERIMENT RESULTS}

To evaluate the performance of the proposed methods, experiments are conducted using signals from three sets of publicly available ECG databases: PTB [32], MIT-BIH [33] and Milano [34]. The PTB database is provided by the National Metrology Institute of Germany and contains 549 records from 294 subjects. The MIT-BIH database contains 48 half-hour excerpts of two-channel ambulatory ECG recordings, obtained from 47 subjects studied by the BIH Laboratory between 1975 and 1979. Each record from this database consists of conventional 12 and 3 leads ECG. The duration of the recordings vary for each subject. Milano database consists of 23 recordings about 30 minutes long, from 23 young normal subjects. Recordings consist of the three orthogonal ECG leads. A concerted effort was made to ensure the maximum fidelity of recordings A subset of 13 subjects was selected to test proposed method. The selected subjects did not exhibit significant arrhythmias. Some of ECG records held in the database contain significant amount of noise and other artifacts which can significantly reduce the valid heartbeat information. Most severely affected records were therefore not used in the performed tests. Since the database only contains one record for each subject, records were partitioned into two halves. The first half was then used as a "training set" and the second half as a "test set" for classification stage. The training and test sets should in the final evaluation of the proposed system be an ECG recordings made on different days for the same subject. The feature matrix for classification is formed of 18 extracted parameters, described in the earlier sections and 14 windows or beats for each subject. By using ECG stream of 8 seconds length with no overlapping, different number of beats are separated into windows where each window has one beat. Several different window lengths tested in this work show approximately the same classification performance as long as full multiple ECG beats are present in the extracted window. Figure 6 shows the scatter plot of extracted parameters including amplitude, time, width and AR coefficients for both parts (training and test) of each ECG subject in the database. Each color represents extracted parameters for individual ECG sample.
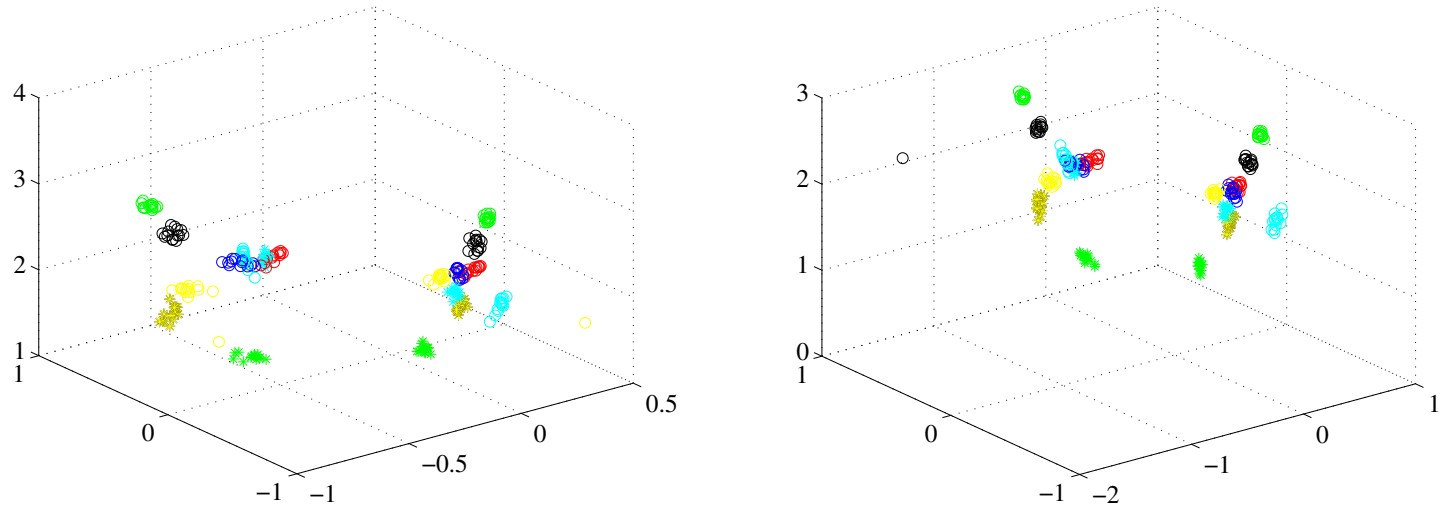

a) Amplitude (training)

Amplitude (test) 

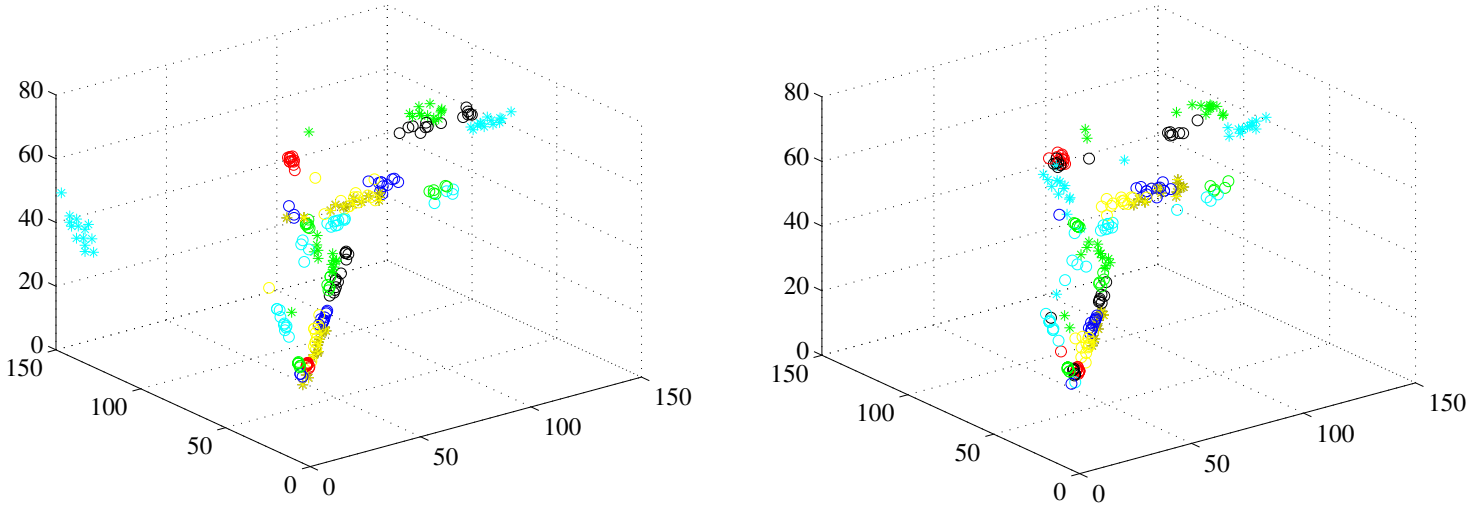

b) Time (training)

Time (test)
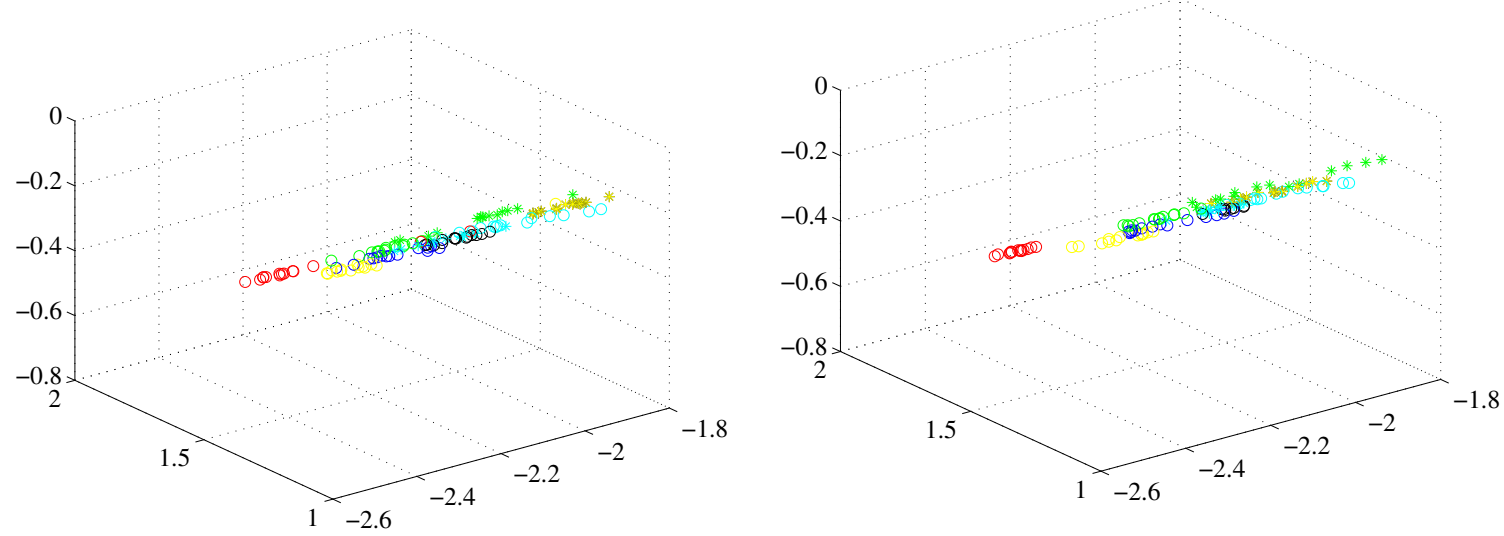

c) $\mathrm{AR}$ (training)

$\mathrm{AR}$ (test) 


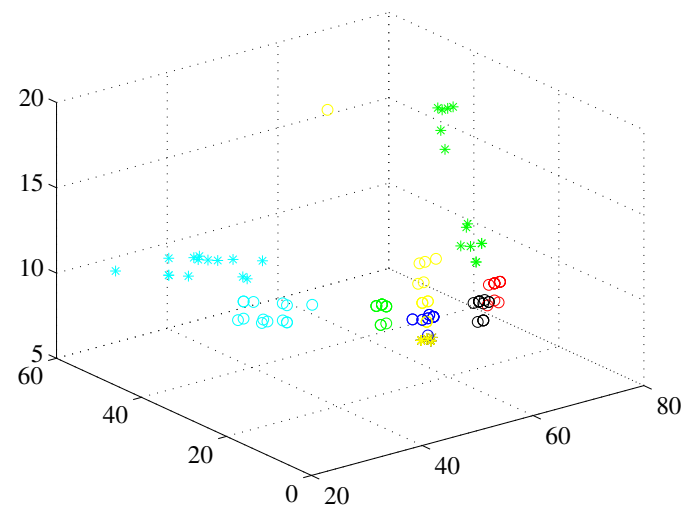

d) Width (training)

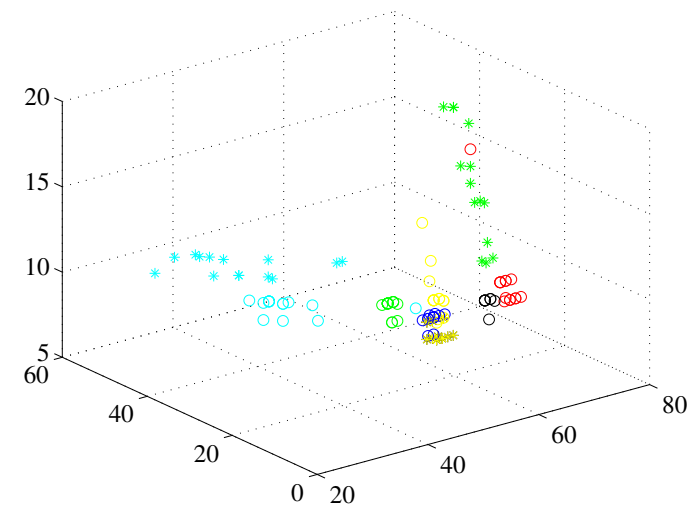

Width (test)

FIGURE 6: Extracted parameters in 3D feature space.

\subsection{Individual Subject Recognition using k-nn}

To evaluate the performance of the proposed system, k-nn classification algorithm has been applied using "knnclassify" function from classification toolbox in Matlab. The k-nearest neighbor is a statistical classification algorithms used for classifying objects based on closest training examples in the feature space. K-nn is a type of instance-based learning, or lazy learning where the function is only approximated locally and all computation is deferred until classification. The k$\mathrm{nn}$ is amongst the simplest of all machine learning algorithms: an object is classified by a majority vote of its neighbors, with the object being assigned to the class most common amongst its $\mathrm{k}$ nearest neighbors. The "knnclassify" function can be called using syntax: " $\mathrm{C}=\mathrm{knn}$ (training, test, classes)". In this case "C" shows the classification factors that express algorithm performance given in Table 2. "Training" and "test" sets are different $8 \mathrm{sec}$ time intervals of each subject and parameter "classes" specifies the number of subjects included in this test. Both training and test sets represent the ECG recordings taken on different days on the same set of subjects. Training set includes 18 extracted parameters and 14 windows or beats for 13 subjects, where test set includes another 18 parameters from other 14 windows or beats for the same 13 subjects.

The obtained results presented in Table 2 show that $100 \%$ accuracy of subject classified can be obtained if all extracted features are used in classification stage. High identification accuracy comes from the matching parameters between the training and test sets of extracted features for the 13 subjects used in this experiment. A high percentage of negative productive values are obtained when smaller number of parameters is used in tests, e.g. amplitude and AR parameters only. Negative productive value indicates the performance of a diagnostic testing procedure. It is defined as the proportion of subjects with some bias compared to the average of other readings that are correctly classified. Table 2 gives the correct identification rate and illustrates comparison of experimental results for different sets of parameters used in training and test sets combination. Suggesting width parameters alone to classify 13 subjects illustrate very low correct rate $(55.56 \%)$. It is important to note that amplitude and AR parameters can reach a high rate of classification (98.89\%) although high percentage of negative test through the procedure is recorded. By using all extracted features (Amplitude, Time, Width and AR model) to identify individual, it is possible to reach $100 \%$ accuracy of classified subjects with a reasonable negative test value. 


\begin{tabular}{c|c|c}
\hline Classification Type & $\begin{array}{c}\text { Correct } \\
\text { Rate }\end{array}$ & $\begin{array}{c}\text { Negative Productive } \\
\text { Value (NP) }\end{array}$ \\
\hline Width & $55.56 \%$ & $33 \%$ \\
\hline Amplitude & $98.89 \%$ & $86 \%$ \\
\hline $\begin{array}{c}\text { Amplitude and AR } \\
\begin{array}{c}\text { Amplitude, Time, Width and } \\
\text { AR model }\end{array}\end{array}$ & $95 \%$ & $87 \%$ \\
\hline
\end{tabular}

TABLE 2: Classification results of biometric system using k-nn.

\subsection{Individual Subject Recognition using SVM}

In addition to high accuracy classification between subjects, further work of Support Vector Machine (SVM) algorithm is applied to estimate classification rate for individual subjects. In order to perform individual recognition of subject, all extracted parameters for single subject are compared with extracted parameter of the same subject and with extracted parameter of another subject. Figure 7 a illustrates no classification between extracted parameters of subject 1 and the extracted parameters of the same subject, as the SVM margin line is lower than all extracted parameters in free space dimension. While, Figure $7 \mathrm{~b}$ shows noticeable classification rate between extracted parameters of subject 1 and the extracted parameters of subject 2, SVM margin line is located between the extracted parameters.

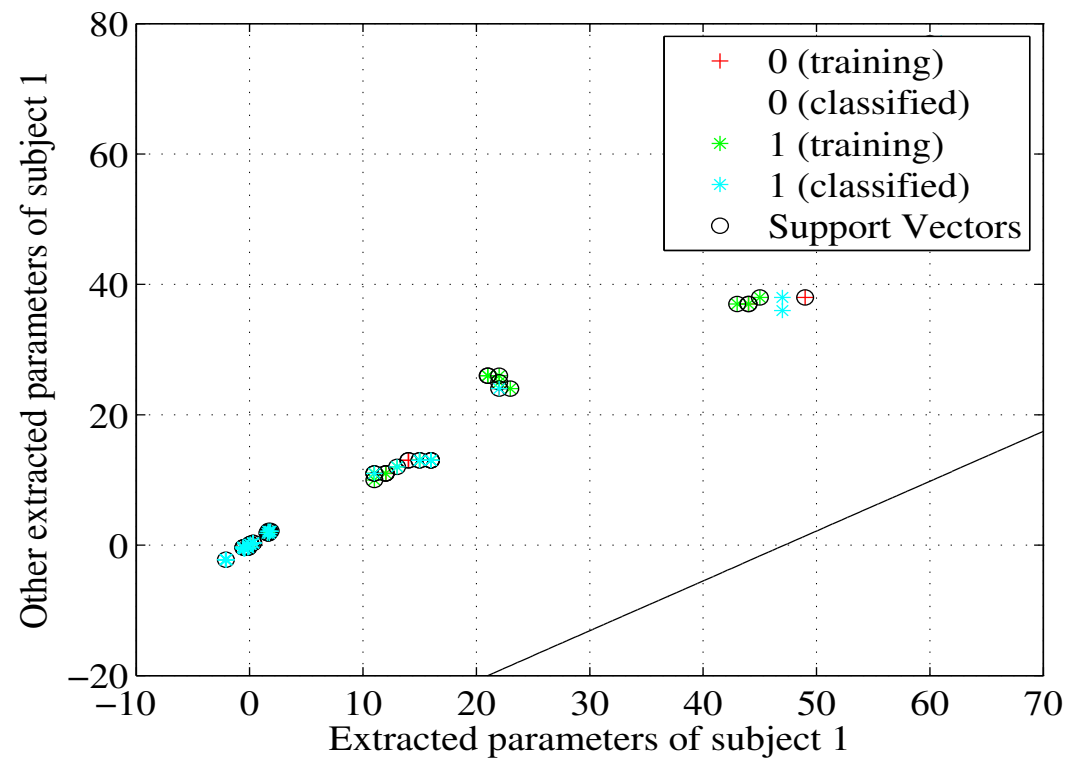

a. Classification results of extracted parameters for the same subject 


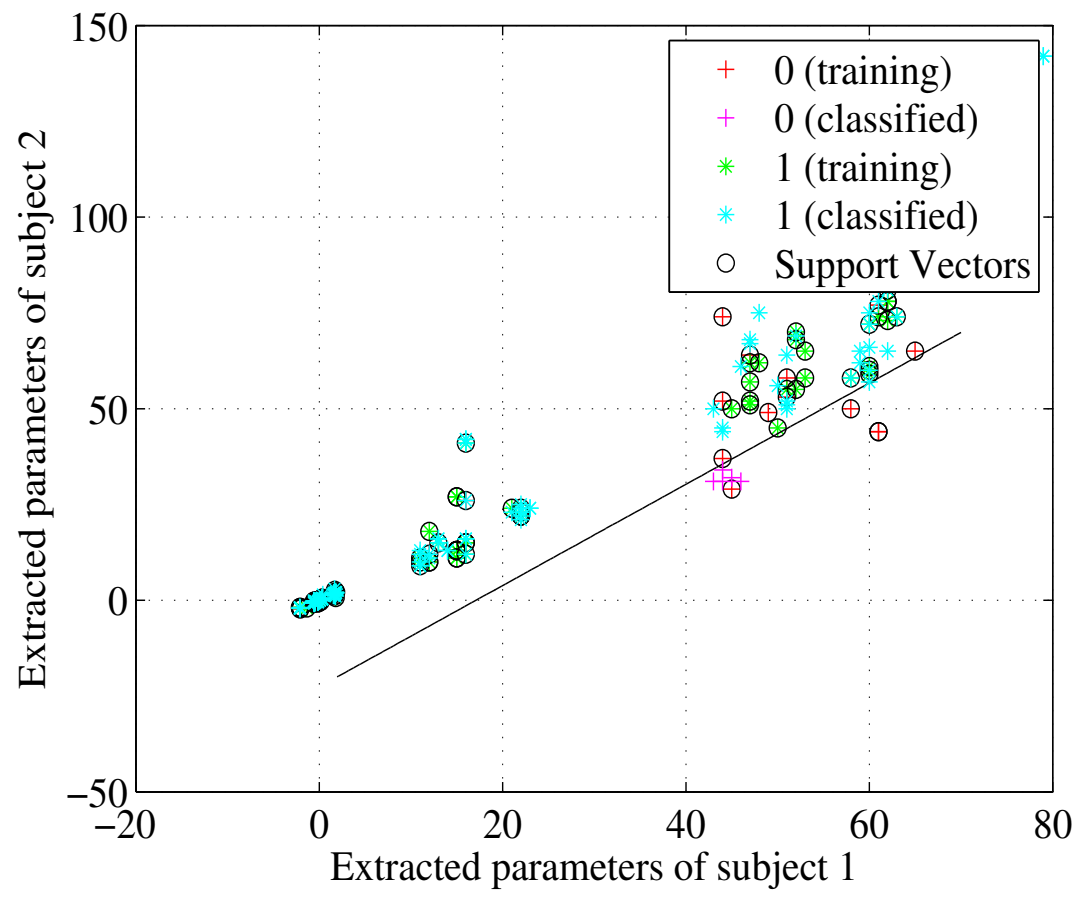

b. Classification results of extracted parameters for two different subjects.

FIGURE 7: SVM classification results of individual subject recognition.

\subsection{Subject Beat Recognition using k-nn and SVM}

In addition to the high classification rate of individual subject recognition using k-nn and SVM, this work investigates the matching and similarity between the extracted parameters from the individual beats of the same and different subjects. Similarity between extracted parameters of two beats of the same subject proves the high classification rate of this approach. In this test, 14 beats from 5 different subjects have been classified using k-nn and SVM to show the matching between the extracted parameters of beats for the same and different subjects. The extracted parameters from the first seven beats of each subject are used as "test" in the k-nn and SVM classification algorithms, while the parameters extracted from the other seven beats are used as "training" set. Table 3 shows correct classification rate between extracted parameters from beats for the same subject using k-nn, while the classification rate is very low between the extracted parameters of different subjects.

\begin{tabular}{|c|c|c|c|c|c|}
\hline Subjects & 1 & 2 & 3 & 4 & 5 \\
\hline 1 & $100 \%$ & $55 \%$ & $54 \%$ & $58 \%$ & $44 \%$ \\
\hline 2 & $55 \%$ & $100 \%$ & $52 \%$ & $56 \%$ & $54 \%$ \\
\hline 3 & $54 \%$ & $52 \%$ & $90.5 \%$ & $48 \%$ & $59 \%$ \\
\hline 4 & $58 \%$ & $56 \%$ & $48 \%$ & $100 \%$ & $57 \%$ \\
\hline 5 & $44 \%$ & $54 \%$ & $59 \%$ & $57 \%$ & $91 \%$ \\
\hline
\end{tabular}

TABLE 3: k-nn classification results of beats for the same and different subjects.

SVM classification algorithm is also used to evaluate the similarity and matching between the extracted parameters of beats for the same subject. Figure 8 shows the similarity and matching of five subjects to identify beat recognition. Results show high similarity and matching between test 
and training data selected for this test and lead SVM margin line to locate out of classification area.
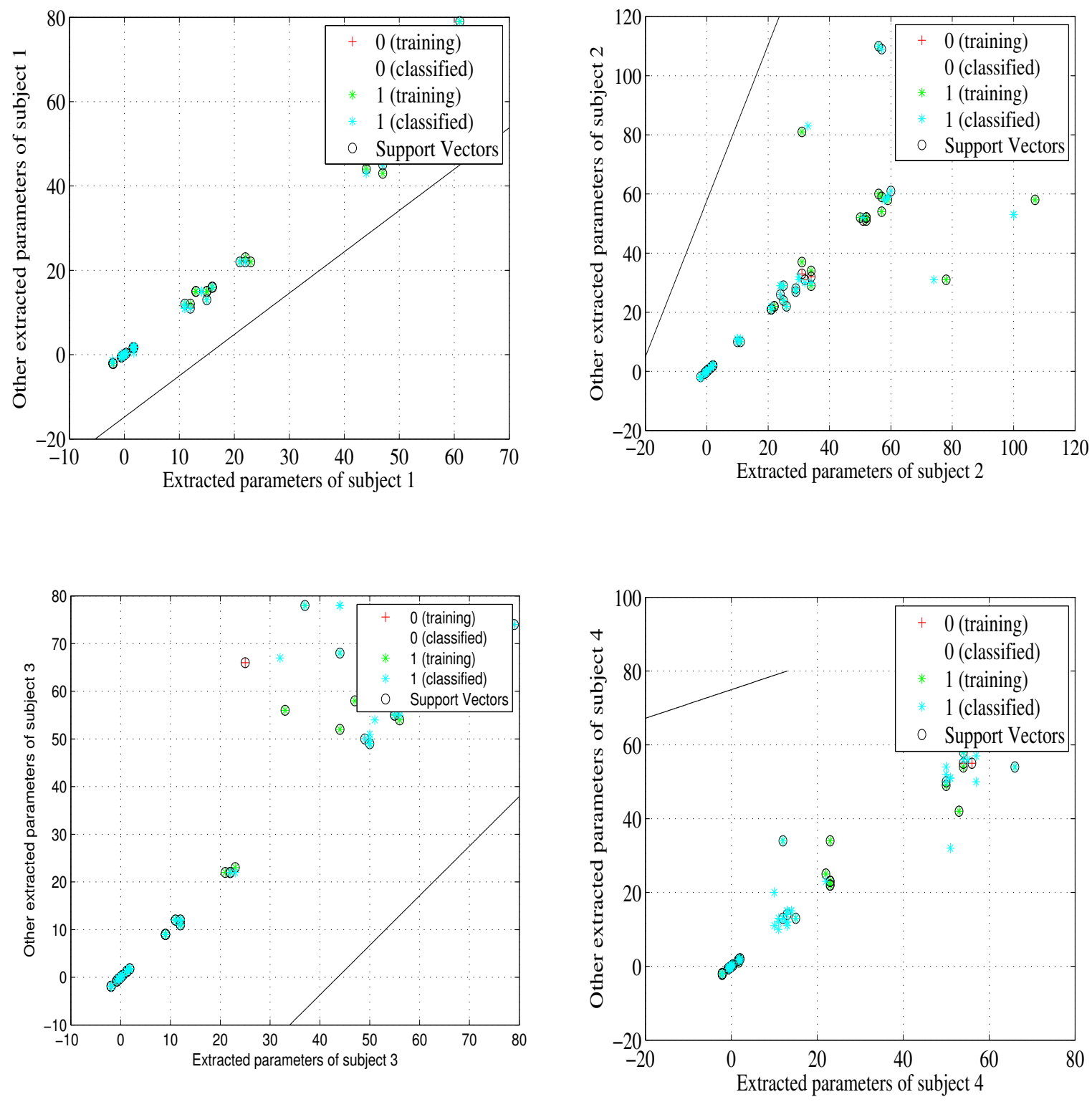


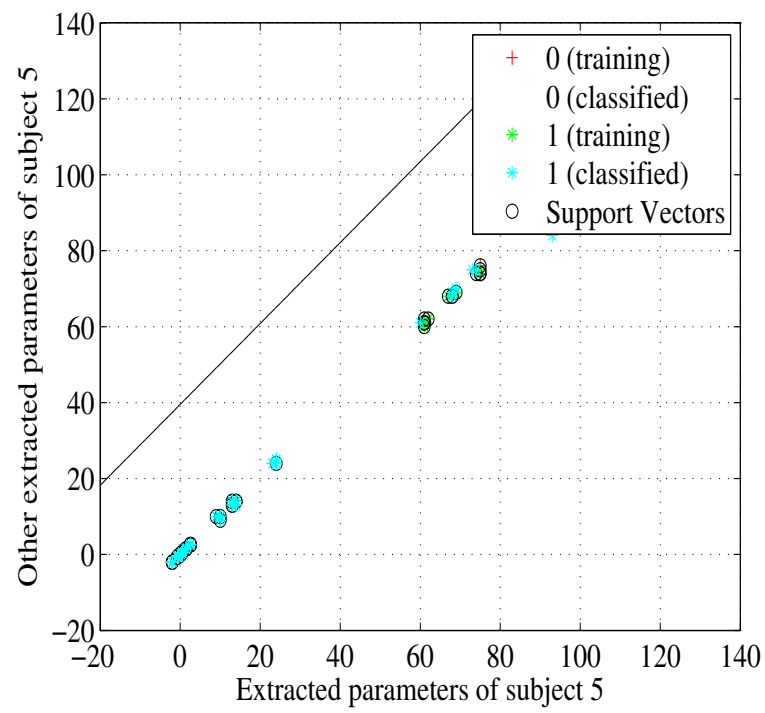

FIGURE 8: Classification results of beats for the same subject using k-nn and SVM.

In order to evaluate the goodness of using AR models to design ECG biometric algorithm, correct classification rate of this method has to be compared with the other correct classification rates of ECG biometric approaches mentioned in the introduction. Table 4 shows that the correct classification rate to identify humans based on their ECG signal using AR model can achieve up to $100 \%$ classification accuracy. This high classification rate is achieved due to the high accuracy of modeling to the ECG signal using AR models, see Figure 5.

\begin{tabular}{|c|c|}
\hline ECG Biometric method & Correct classification rate \% \\
\hline Wavelet transform & 98 \\
\hline $\begin{array}{c}\text { Kohonen self-organizing maps (SOM) and } \\
\text { Learning Vector Quantization (LVQ) }\end{array}$ & 94 \\
\hline $\begin{array}{c}\text { Artificial Neural Networks (ANN) and Linear } \\
\text { Discriminant Analysis (LDA) }\end{array}$ & 95 \\
\hline Hidden Markov Model (HMM & 100 \\
\hline Autoregressive (AR) model & 85 \\
\hline
\end{tabular}

TABLE 4: Evaluation table of correct classification for different ECG biometric methods.

The ability to build accurate ECG biometric system using modeling techniques depends on the accuracy to model ECG signal. Autoregressive Moving Average (ARMA) model could be investigated as an extraction approach for more advanced methods of modeling to ECG signal. The extracted parameters from applied ARMA model could be used in the classification algorithm in order to achieve high correct rate of classification. 


\section{CONCLUSIONS}

A biometric system for automatic analysis of a single lead electrocardiogram (ECG) for human identification is presented in this work. The first stage of this system consists of a band-pass Butterworth filter used to remove noise and other artifacts present in the raw ECG signal. The $R$ peaks of an ECG stream are then localized by filtering a signal through the bank of filters and analysing each filtered signal component individually. The individual heart beats of an ECG record are aligned along the $R$ peak position and truncated by a window size based on the heart rate variability (HRV) of individuals' ECG. The temporal, amplitude, width and AR parameters extracted from each ECG signal are used to identify individual ECGs. An approach of modeling two or more successive ECG beats, using a discrete form of an autoregressive (AR) signal model of order $p$ is applied to extract AR modeling features, $\operatorname{AR}(p)$. A subset of 13 subjects was selected in this work in order to test proposed system. All the extracted parameters for each subject are fitted into k-nn and SVM classification algorithm to classify and eventually identify "test" parts of each signal. $100 \%$ identification accuracy for 13 different ECG recordings has been achieved in this project using k-nn and SVM classification algorithms, when each ECG signal is split into "train" and "test" section. Results presented in this work indicate that the ECG based system might lead to an accurate biometric identification in some practical applications.

\section{REFERENCES}

[1] Y. L. Singh and P. Gupta (2008), ECG to Individual Identification, Biometrics: Theory, Applications and Systems, 2008. BTAS 2008. 2nd IEEE International Conference on, pp. 1 8.

[2] S.-H. Lin (2000), An Introduction to Face Recognition Technology, Informing Science special Issue on Multimedia Informing Technologies, vol. 3, no. 1, pp. 1-7.

[3] Y. Wang, F. Agrafioti and D. Hatzinak (2008), Analysis of Human Electrocardiogram, Hindawi Publishing Corporation, EURASIP Journal on Advances in Signal Processing, pp. 11.

[4] T. Shen and W. Tompkins (2002), One-lead ECG for identity verification, in: Proceedings of the 24th Annual International Conference of IEEE EMBS, pp. 62-63.

[5] K. Jain, A. Ross and S. Prabhakar (2004), An introduction to biometric recognition, IEEE Transactions on Circuits and Systems for Video Technology, vol. 14, no. 1, pp. 4-20.

[6] R. Hoekema and G. J. H. Uijen (2001), Geometrical aspects of the interindividual variability of multilead ECG recordings, IEEE Transactions on Biomedical Engineering, vol. 48, no. 5, pp. 551-559.

[7] I. Eisenstein, J. Edelstein and R. Sarma (1982), The electrocardiogram in obesity, J. Electrocardiol., vol. 15, no. 2, pp. 115-118.

[8] S. Safie, J. Soraghan and L. Petropoulakis (2011), ECG biometric authentication using Pulse Active Width (PAW), Biometric Measurements and Systems for Security and Medical Applications (BIOMS), 2011 IEEE Workshop on, pp. 1 - 6.

[9] I. Odinaka, A. Kaplan, J. O'Sullivan and L. Po-Hsiang (2012), ECG Biometric Recognition: A Comparative Analysis, IEEE Transactions on Information Forensics and Security, vol. 7, no. 6, pp. 1812 - 1824. 
[10] L. Biel, O. Pettersson, L. Philipson and P. Wide (2001), ECG analysis: A new approach in human identifications, IEEE Trans. Instrum. Meas., vol. 50, no. 33, pp. 808-812.

[11] M. Kyosoand and A. Uchiyama (2001), Developmentofan ECG identification system, in Proc. 23rd Ann. EMBS Int. Conf.

[12] J. Irvine, B. Wiederhold, L. Gavshon and S. Israel (2001), Heart rate variability: A new biometric for human identification, Proc. Int. Conf. Artificial Intelligence (ICAI 2001).

[13] S. Mahmoodabadi, A. Ahmadia and M. Abolhas (2005), ECG Feature Extraction using Daubechies Wavelets, Proceedings of the fifth IASTED International conference on Visualization, Imaging and Image Processing, pp. 343-348.

[14] P. Tadejko and W. Rakowski (2007), Mathematical Morphology Based ECG Feature Extraction for the Purpose of Heartbeat Classification, 6th International Conference on Computer Information Systems and Industrial Management Applications, CISIM '07, pp. 322327.

[15] C. Alexakis, H. O. Nyongesa, R. Saatchi and N. D. Har (2007), Feature Extraction and Classification of Electrocardiogram (ECG) Signals Related to Hypoglycaemia, Conference on computers in Cardiology, pp. 53.

[16] P. Hee-Soo and W. Soo-Min (2009), ECG Pattern Classification Based on Generic Feature Extraction, Proceedings of the 3rd WSEAS Int. Conf. on circuits, systems, signal and telecommunication, (CISST'09) ISSN:, vol. 21.

[17] W. Liang, Y. Zhang and J. Tan (2009), A Novel Approach to ECG Classification Based upon Two-Layered HMMs in Body Sensor Networks, Multidisciplinary Digital Publishing Institute, vol. 14, pp. 5994-6011,.

[18] B. Vuksanovic and M. Alhamdi (2013), ECG Based System for Arrythmia Detection and Patient Identification, ITI2013, pp. 1-7.

[19] D. Gari, A. Francisco and M. Patrick (2006), Biomedical Engineering. :Advanced Methods And Tools for ECG Data Analysis, Artech House, Inc.

[20] I. Duskalov (1998), Developments in ECG acquisition, preprocessing, parameter measurement, and recording, IEEE Engineering in Medicine and Biology Magazine, vol. 7, no. 2 , pp. 50 - 58.

[21] A. Ziarani and A. Konrad (2010), Non linear Adaptive method of elimination of power line interference in ECG signals, IEEE Transactions on Biomedical Eng, vol. 49, no. 6, pp. 540544.

[22] S. Pooranchandra and N. Kumaravel (2010), A novel method for elimination of power line frequency in ECG signal using hyper shrinkage functions, Digital Signal Processing, vol. 18, no. 2 , pp. 116-126. 
[23] S. Dhillon and S. Chakrabarti (2001), Power Line Interference removal From Electrocardiogram Using A Simplified Lattice Based Adaptive IIR Notch Filter, Proceedings of the 23rd Annual EMBS International conference,Istanbul, Turkey.

[24] M. Chavan, R. Aggarwala and M. Uplane (2008), Interference reduction in ECG using digital FIR filters based on Rectangular window, WSEAS Transactions on Signal Processing, vol. 4, no. 5 , pp. 340-49.

[25] S. K. Jagtap (2012), The impact of digital filtering to ECG analysis: Butterworth filter application, Communication, Information \& Computing Technology (ICCICT), 2012 International Conference on, pp. 1-6.

[26] H.-Y. Zhou and K.-M. Hou (2008), Embedded real-time QRS detection algorithm for pervasive cardiac care system, 9th International Conference on Signal Processing, pp. 2150 - 2153.

[27] J. F. Moraes (2002), QRS complex detection algorithm using electrocardiogram leads, Computers in Cardiology, pp. 205 - 208.

[28] G. Kokturk (1998), A real-time simulated QRS detection system constructed using wavelet filtering technique, IEEE-SP International Symposium on Time-Frequency and Time-Scale Analysis, pp. $281-284$.

[29] N. Uchaipichat and S. Inban (2010), Development of QRS Detection using Short-time Fourier Transform based Technique, IJCA Journal.

[30] S. A. Israel, J. M. Irvine and A. Cheng (2005), ECG to identify individuals, Pattern Recognition, pp. 133-142.

[31] C. Anderson, E. Stolz and S. Shamsunder (1995), Discriminating Mental Tasks Using EEG Represented by AR Models, Engineering in Medicine and Biology Society, IEEE 17th Annual Conference, vol. 2.

[32] A. Goldberger, L. Amaral, L. Glass and Hausdorff (2000), Components of a New Research Resource for Complex Physiologic Signals., PhysioBank, PhysioToolkit, and PhysioNet:, vol. 23, no. 21, pp. 101.

[33] R. Mark (1990), The MIT-BIH Arrhythmia Database on CD-ROM and software for use with it, IEEE in Computers in Cardiology 1990, Proceedings.

[34] D. Milano (1992), Politecnico Biosignals Archives on CD-ROM, (Copyright (C) Politecnico. 1992). 